Revue internationale de l'économie sociale

Recma



\title{
Temps forts
}

\section{Maryline Filippi, Michel Abhervé et Eric Bidet}

Numéro 338, octobre 2015

URI : https://id.erudit.org/iderudit/1033868ar

DOI : https://doi.org/10.7202/1033868ar

Aller au sommaire du numéro

\section{Éditeur(s)}

Association Recma

ISSN

1626-1682 (imprimé)

2261-2599 (numérique)

Découvrir la revue

Citer ce document

Filippi, M., Abhervé, M. \& Bidet, E. (2015). Temps forts. Revue internationale de l'économie sociale, (338), 7-13. https://doi.org/10.7202/1033868ar d'utilisation que vous pouvez consulter en ligne.

https://apropos.erudit.org/fr/usagers/politique-dutilisation/ 


\section{TEMPS FORTS}

\section{La conférence recherche de l'ACI : Paris 2015}

L'Alliance coopérative internationale (ACI) a souhaité organiser sa conférence de recherche annuelle sur le thème « Futur du modèle coopératif: créativité, innovation et recherche ", à Paris, du 27 au 30 mai 2015. Cet événement a associé de façon originale, d'une part, Coop FR, le Crédit coopératif, Coop de France et toutes les familles de la coopération et, d'autre part, l'Institut des sciences et industries du vivant et de l'environnement (AgroParisTech), l'Institut national de recherche agronomique (Inra) et ses départements « Sciences action développement " et "Activités produits territoires " (SAD-APT), avec la participation de l'Essec Business School. Il a accueilli plus de cent cinquante participants, de trente-quatre pays, au cours de vingt ateliers sur des thèmes aussi divers que la gouvernance, les coopératives de travailleurs, les nouvelles formes de coopération, les innovations sociales, etc. Le succès de cette conférence est dû non seulement au nombre de participants, mais aussi à leur diversité scientifique et à la qualité des débats, des conférenciers et des participants aux tables rondes.

Parmi les temps forts, l'Organisation des Nations unies pour l'alimentation et l'agriculture (FAO) a donné la primeur de son rapport « The State of Food Insecurity in the World 2015 », qui souligne la réduction de la pauvreté en célébrant l'action positive des coopératives; Jean-Louis Bancel, président du Crédit coopératif, a dévoilé la nouvelle formulation des principes de l'ACI (The New Guidance Notes on Cooperative Principles) ${ }^{(1)}$. La conférence inaugurale du 28 mai a été assurée par le professeur Michael L. Cook, de l'université du Missouri-Columbia,

(1) Ica.coop/sites/default/files/attachments/FR Notes d'orientation - Consultation 2015-05.pdf. sur le thème "Coopératives agricoles: challenges et opportunités pour un monde meilleur", donnant une présentation stimulante des questions de recherche ouvertes également aux praticiens. Elle a permis d'introduire la première table ronde: "Agriculture, alimentation durable et systèmes de distribution: quelles perspectives? Enjeux pour les coopératives.» La deuxième, qui a suivi la conférence de Jean-Louis Bancel, était dédiée aux valeurs coopératives $^{(2)}$.

\section{Principal résultat: le pouvoir des coopératives s'accroît}

Les études internationales menées par les analystes, les chercheurs et les praticiens de tous les secteurs d'activité (commerce, banque, agriculture, etc.) ont contribué à démontrer que non seulement les coopératives étendent leur pouvoir, mais également que leur efficacité s'affirme de plus en plus: pour réduire la pauvreté ou le gaspillage alimentaire, pour donner un revenu décent via un accès aux marchés ou à un emploi, pour rendre accessible au plus grand nombre l'éducation, la santé, le logement, la culture. Répondre aux défis actuels nécessite cependant d'améliorer la compréhension des processus mobilisés dans la compétition économique (le cycle de vie des coopératives agricoles, par exemple), d'identifier les indicateurs pour proposer des mesures appropriées pour un soutien plus efficace des politiques publiques et de développer de nouveaux outils et de nouvelles mesures de la solidarité, de l'engagement et de la performance au service des coopérateurs. En contrepartie des succès économiques et sociaux, les transformations des coopératives soulignent effectivement le besoin

(2) Le programme complet et la liste des intervenants sont consultables sur le site de la conférence: Ica-paris2015. com/index.php/program. 
de réfléchir aux façons dont les coopératives combinent compétitivité et satisfaction des membres dans le respect des valeurs et des principes coopératifs.

Les débats ont donc été extrêmement denses, associant travaux théoriques et approches pratiques, avec des expériences en provenance des pays du Nord comme du Sud, d'Asie et d'Afrique, mobilisant l'ensemble des activités: l'agriculture, la banque, l'éducation, le commerce ou l'énergie.

Le constat a été fait que, si les coopératives deviennent de plus en plus performantes, elles sont également plus complexes dans leur organisation. Dès lors, des pistes de réflexion se sont dégagées autour des notions d'identité, d'équilibre et de durabilité, que nous allons présenter.

\section{Coopératives et caractérisation de l'identité}

Quelle est la nature d'une coopérative? Soulignée par le professeur Cook, cette question, faussement simple, nécessite de bien définir ce qu'est une coopérative afin d'en mesurer la performance économique en comparaison avec les autres formes d'entreprises, mais, surtout, afin de créer des indicateurs de mesure appropriés pour cerner sa réelle plus-value et son originalité. L'histoire des coopératives révèle une très grande hétérogénéité des formes organisationnelles: petites coopératives avec peu de membres, groupes coopératifs, internationalisés, avec des formes hybrides ou dans les services. Comment survivre? Comment respecter les principes coopératifs, lorsque les membres partagent le capital de la coopérative avec des non-membres ou que les associés se dénombrent en milliers?

La gouvernance est un point névralgique qui a été abordé dans de nombreuses communications sous différents angles: qui possède, qui décide et qui contrôle? Plusieurs communications ont évoqué les questions de l'identité et de l'organisation, avec différentes parties prenantes, le besoin de repenser les relations entre les membres, d'adopter une perspective multidisciplinaire pour saisir leur complexité avec des outils pour le diagnostic, la décision et l'organisation. Le défi de trouver un ou des modèles de gouvernance coopérative permettant de garantir l'exercice légitime du droit de propriété des associés coopérateurs, dans les petites coopératives comme dans les groupes, a été avancé comme un enjeu majeur.

\section{Coopératives et recherche d'équilibre (balance)}

Certaines communications ont insisté sur le fait que les coopératives sont des entreprises avec des valeurs humaines et responsables, visant à apporter de meilleurs revenus, à contribuer au développement des espaces ruraux, à proposer des nouveaux services (" coopératives de services » aux Etats-Unis ou nouvelles coopératives dans les pays de l'Est) ou à permettre un épanouissement de leurs membres. L'opposition initiale entre les objectifs économiques et sociaux se transforme ainsi en questionnement autour de la capacité des coopératives à associer ces deux dimensions pour être davantage en adéquation avec les besoins de leurs associés coopérateurs. La question de l'hétérogénéité des membres a été l'une des plus abordées, étant donné que les coopératives ne sont pas des entreprises de capitaux, mais de personnes. Aussi cette hétérogénéité est-elle susceptible d'accroître les conflits dans la prise de décision qui repose sur le principe démocratique de "Un individu égale une voix » et, en conséquence, d'amoindrir sa capacité à prendre en compte l'intérêt de chacun des membres.

Accéder aux marchés souligne l'importance de sécuriser l'organisation des chaînes alimentaires, d'équilibrer les pouvoirs de négociation entre les différentes parties prenantes, de renforcer le pouvoir des producteurs face aux acteurs de la grande distribution et aux multinationales dans la transformation et le commerce. 
Les spécificités du modèle quant aux relations entre associés coopérateurs, dirigeants et employés doivent être identifiées pour diriger une coopérative comme une entreprise, dans un "esprit coopératif » et avec une responsabilité sociale, afin de renforcer la capacité de résilience qu'elles ont démontrée lors des dernières crises financières ou alimentaires.

\section{Coopératives et durabilité ou soutenabilité}

Dans un monde en mutation, les coopératives ont constamment besoin de s'adapter et de renouveler leurs frontières. Elles ne sont pas seulement des entreprises, mais proposent aussi un mode de coordination spécifique et un état d'esprit. Elles sont différentes des entreprises de droit commercial dans leur objectif, mais aussi au regard de la satisfaction de leurs associés coopérateurs dans une perspective de moyen et long termes pour les générations futures. Les coopératives ont besoin d'innover afin de relever des défis comme celui du genre et de se confronter à des enjeux, la pression foncière et le gaspillage alimentaire, par exemple.

La diversité est une source de richesse dont les membres et les employés peuvent tirer avantage au lieu de traduire leur hétérogénéité en conflits. Partager les expériences à l'échelle internationale des secteurs d'activité et des organisations a ouvert le monde des possibles pour créer de nouvelles solutions et trouver les moyens de répondre non seulement aux besoins élémentaires des associés coopérateurs, mais aussi aux besoins sociaux et culturels, motivations essentielles pour respecter les principes et les valeurs coopératives. Cette conférence de recherche a aussi souligné la nécessité de communiquer sur les incroyables pouvoirs des coopératives et sur la spécificité de leur modèle.

\section{Principale recommandation aux décideurs : développer la recherche et l'éducation coopératives}

Pourquoi la recherche est-elle importante? Spécifier l'identité du modèle coopératif offre un meilleur chemin pour l'action. Les intervenants ont convergé sur le besoin de renforcer la recherche académique afin d'adapter le modèle d'entreprise coopératif pour être plus compétitif avec des valeurs coopératives. "Construire un capital coopératif dirigé par un esprit coopératif! » Les coopératives sont des entreprises pragmatiques, qui combinent des dimensions sociales et économiques, mais elles ne sont pas des organisations idéologiques.

Pourquoi l'éducation est-elle essentielle? C'est une voie pour créer un monde meilleur, plus respectueux de tous. Il est nécessaire d'améliorer l'éducation et la formation des associés coopérateurs, des dirigeants et des employés: pour les nouvelles générations, pour les femmes, afin de réduire la pauvreté et de renforcer les communautés. Les défis sont immenses pour les coopératives et peuvent leur donner l'occasion de révéler leur potentiel. Les intervenants ont signalé la nécessité de promouvoir les coopératives et l'entreprenariat coopératif en réponse aux enjeux internationaux tels que le changement climatique, l'alimentation durable, la démocratie. Enfin, tous ont insisté sur le besoin de caractériser le modèle coopératif, l'objectif étant de mieux comprendre pour agir et de développer des formations à l'international pour apprendre de l'expérience des autres, de leurs différences et de leurs similitudes. Le constat a été fait qu'il nous faut, tous ensemble, œuvrer à ce monde meilleur.

Pourquoi devons-nous combiner recherche et éducation? Il n'y a pas d'action sans conscience. Abandonner les principes coopératifs peut se révéler dangereux. Le défi est de repenser le modèle coopératif sans le dévoyer ni perdre l'âme des coopératives.

Tels ont été les sujets débattus pour relier les chercheurs et les praticiens autour des enjeux du "futur du modèle coopératif ».

MARYLINe Filippi (COMITÉ d'ORganisation DE LA CONFÉRENCE RECHERCHE DE L'ACI, PARIS, 2015) 


\section{Les XVes rencontres du RIUESS, à Reims}

Les $\mathrm{XV}^{\text {es }}$ Rencontres interuniversitaires de l'économie sociale et solidaire (RIUESS) étaient organisées du 27 au 29 mai 2015 par le laboratoire d'économie et de gestion RegardS-EA 6292 de l'université de ReimsChampagne-Ardennes, en collaboration avec Neoma Business School, sur le thème « La créativité de l'économie sociale et solidaire est-elle soluble dans l'entrepreneuriat?". Cet événement a vu la montée en puissance de la participation des doctorants, mettant en évidence le nombre croissant de jeunes chercheurs qui travaillent dans diverses disciplines sur l'économie sociale et solidaire (ESS) et contribuent à une meilleure connaissance de celle-ci. Cela pose la question de la valorisation de ces travaux, mais aussi celle du devenir de ces chercheurs confirmés confrontés à la faiblesse des postes dans l'enseignement supérieur et la recherche.

Le texte des communications, concernant la gestion démocratique, les pôles territoriaux de coopération économique, l'innovation, le vivre ensemble, la gestion, les frontières de l'ESS, le renouveau des pratiques coopé-ratives, l'institutionnalisation de l'ESS et bien d'autres sujets, est consultable sur le site du RIUESS ${ }^{(3)}$. Notons en particulier une communication de Laurent Fraisse, Laurent Gardin, Jean-Louis Laville, Francesca Petrella et Nadine Richez-Battesti, qui a éclairci le concept d'entrepreneuriat social dans le contexte français, tel qu'il a été défini dans le cadre du projet Emes (International Research Network) sous l'impulsion de Jacques Defourny. Elle a souligné un risque pour l'ESS «quand l'entrepreneuriat social est rabattu sur le social business et sa rhétorique de l'autofinancement sur le marchéqui a déjà conduit à une dérive du microcrédit ». Ce rendez-vous annuel témoigne, d'une part, de l'augmentation du nombre d'universités

(3) Socioeco.org/bdf_colloque-11_fr.html. françaises investies dans des recherches relatives à l'ESS et organisant des formations au niveau licence et master et, d'autre part, de l'intérêt d'un lieu d'échanges associant un certain nombre d'universités étrangères. Les XVI ${ }^{\text {es }}$ Rencontres auront lieu à Montpellier les 25,26 et 27 mai 2016 et auront pour thème "Les “communs" et l'économie sociale et solidaire: une identité commune? ", initié lors des rencontre de Reims au sein d'un atelier "ESS et bien commun ". L'appel à communications sera lancé prochainement.

MICHEL ABHERVÉ

\section{Le prix de thèse de l'Addes 2015}

Le jury du prix de thèse de l'Association pour le développement de la documentation sur l'économie sociale (Addes), a reçu cette année dix-huit thèses ${ }^{(4)}$. Lors de sa précédente édition (2013), il en avait reçu huit. Cette croissance à trois chiffres témoigne tout à la fois de la réussite du prix de l'Addes, lancé il y a presque trente ans, et de la reconnaissance de l'économie sociale et solidaire (ESS) en tant qu'objet de recherche de plus en plus pertinent au sein de l'université - et même au-delà, puisqu'un tiers de ces travaux en dépasse le cadre strict.

Pour départager les nombreux travaux qu'il n'était pas en mesure de lire dans leur intégralité, le jury s'est d'abord appuyé sur les rapports de soutenance et sur quelques éléments fondamentaux de chaque thèse, afin de réaliser une première sélection visant à retenir trois " finalistes » pour l'obtention du prix de l'Addes. Sélectionner trois thèses parmi dix-huit n'a pas été sans difficulté, et des travaux de grande qualité ont été écartés à ce stade. Parmi ceux-ci, le jury a particulièrement remarqué la thèse de droit comparé et de droit européen d'Iris Barsan, portant sur le statut juridique de la société coopérative européenne. Comme le souligne

(4) La remise du prix des mémoires de master 2 a fait l'objet d'un rapport dans la rubrique « Temps forts » du numéro 337 de la Recma, page 12. 
le rapport de soutenance, le travail réalisé devrait, à n'en pas douter, déboucher sur un ouvrage de référence en matière de société coopérative européenne.

Le précédent prix de l'Addes avait fait la part belle à l'histoire. Ce sont cette fois-ci des travaux en droit, en sociologie, en économie et en gestion qui ont retenu l'attention du jury. Et on ne peut que se réjouir que l'ESS suscite des travaux de grande qualité dans des disciplines aussi nombreuses et selon des approches aussi variées. On notera d'ailleurs que, sur les dix-huit jeunes docteurs qui ont concouru pour le prix de l'Addes, cinq au moins sont déjà titularisés en université, ce qui montre que l'économie sociale émerge progressivement comme un sujet d'étude pertinent et porteur parmi les principales sciences sociales. Cela témoigne également des progrès accomplis dans la construction d'une communauté scientifique ayant pour objet l'économie sociale, à laquelle l'Addes s'attache depuis plus de trente ans. Selon le procédé appliqué par le jury, les trois thèses retenues sont celles de Delphine Pouchain, de Diane Rodet et de Pascale Chateau-Terrisse.

La thèse de Delphine Pouchain, intitulée "Commerce équitable et prix juste », est une thèse de sciences économiques codirigée par Olivier Favereau (université Paris-Ouest) et Patrick Mardellat (université Lille-2). L'idée centrale défendue est que le modèle du commerce équitable incarne la «bonne économie » au sens d'Aristote, c'est-à-dire une économie garante de la justice dans l'échange, ce que l'on appelle la «justice commutative». L'une des grandes qualités de ce travail, très agréable à lire, est justement de soutenir une thèse au sens propre, au moyen d'une argumentation rigoureuse, dont la progression logique et justifiée amène l'auteure à mobiliser un large éventail de travaux relevant de disciplines très variées (économie, gestion, sociologie, philosophie, anthropologie, sciences politiques). Par ailleurs, cette thèse rappelle que l'histoire de la pensée économique (HPE) est utile pour comprendre des phénomènes économiques très contemporains et qu'il est encore possible d'écrire une thèse d'économie sans y faire figurer une seule équation. Le jury a cependant regretté que l'auteure n'ait pas véritablement abordé la question de la contradiction fondamentale entre le commerce équitable, qui s’inscrit dans la logique de la mondialisation, et les circuits courts, qui valorisent une économie de proximité, ni cherché à étendre sa réflexion à l'ESS pour évaluer si le modèle de celle-ci peut être considéré comme un prolongement - ou une incarnation - de la bonne économie d'Aristote.

Diane Rodet a soumis une thèse de sociologie intitulée "Une production engagée: sociologie des labels, chartes et systèmes participatifs de l'économie solidaire ", qui a été soutenue au Conservatoire national des arts et métiers (Cnam) sous la direction d'Antoine Bevort. Son travail porte sur les dispositifs de qualité propres à l'économie solidaire. L'idée centrale que défend l'auteure est que, dans l'économie solidaire, ces dispositifs de qualité ne sont pas seulement (ni principalement) orientés vers les consommateurs pour les rassurer, mais sont aussi, et avant tout, des éléments destinés à construire ou à renforcer une identité collective. Le jury a tenu à souligner l'excellence de cette thèse, bien construite et bien écrite, sur un sujet apparu en sociologie dans les années 90 avec les travaux fondateurs de Lucien Karpik, mais essentiellement abordé du point de vue de la sociologie de la consommation et encore très peu souvent relié à l'ESS. La réflexion menée s'appuie tout à la fois sur une très bonne connaissance des travaux théoriques en sociologie et en économie et sur un travail empirique portant sur douze réseaux d'économie solidaire, allant des régies de quartier aux associations pour le maintien d'une agriculture paysanne (Amap), en passant par les systèmes d'échanges locaux, le commerce équitable ou la finance solidaire. Le jury a émis quelques réserves, cependant, 
sur le parti pris d'isoler au sein de l'ESS un ensemble d'organisations que l'auteure rassemble sous l'appellation "économie solidaire ». Il s'est également interrogé sur la pertinence de mettre sur le même plan des démarches aussi variées et d'appliquer la même grille d'analyse à des produits et à des organisations.

La thèse de Pascale Terrisse, intitulée « Les outils de gestion, transporteurs et régulateurs des logiques institutionnelles: cas de deux organisations de capital-risque solidaire ", est une thèse de sciences de gestion dirigée par Muriel Jougleux (université Paris-Est). Elle décrit l'évolution du secteur du capital-risque solidaire en France à travers l'étude en deux dispositifs de ce secteur: le Fonds Afrique, mis en place par Garrigue en 2005, et la société FinanCités, créée par Planet Finance en 2007. L'originalité de la démarche est d'analyser les transformations de ces organisations par l'étude des outils de gestion utilisés. Cette recherche porte sur une question essentielle pour l'ESS: celle de la tension entre des logiques institutionnelles distinctes et quelquefois contradictoires. Elle offre par ailleurs une description fine de la structuration et des transformations dans le champ de la finance solidaire.

\section{Tableau 1}

Répartition par type d'établissement

\begin{tabular}{|l|c|}
\hline Type d'établissement & Nombre de thèses \\
\hline Universités & 12 \\
\hline EHESS* $^{*}$ & 2 \\
\hline Cnam $^{* *}$ & 2 \\
\hline $\begin{array}{l}\text { Grandes écoles } \\
\text { (HEC***, SupAgro****) }\end{array}$ & 2 \\
\hline
\end{tabular}

${ }^{*}$ Ecole des hautes études en sciences sociales.

** Conservatoire national des arts et métiers.

*** Hautes études commerciales.

**** Institut national d'études supérieures agronomiques de Montpellier.
Après de nombreux échanges, c'est à cette thèse que le jury de l'Addes a décidé d'attribuer son prix. En couronnant le travail de Pascale Terrisse, il a souhaité tout d'abord récompenser l'originalité d'un sujet de thèse d'un grand intérêt dans l'optique de la loi ESS. Le jury salue ensuite la rigueur théorique, l'honnêteté méthodologique et le potentiel heuristique de cette thèse. Il a particulièrement apprécié la réflexion théorique menée à partir de l'approche néo-institutionnaliste, ainsi que le chapitre méthodologique consacré au logiciel Alceste et la description fine qui est faite des transformations dans le champ de la finance solidaire. Tout en suggérant que l'auteure aurait pu élargir sa perspective théorique en mobilisant des auteurs de la sociologie des organisations comme Mintzberg, Crozier ou Boltanski et Thévenot, le jury a estimé que le travail réalisé était déjà considérable et qu'il pouvait s'agir là de pistes à envisager pour prolonger la réflexion entreprise.

Quelques statistiques concernant la répartition des thèses reçues par type d'établissement sont présentées dans le tableau 1 , par discipline universitaire dans le tableau 2, par objet de recherche dans le tableau 3 et par zone géographique dans le tableau 4 (voir ci-dessous et en page suivante). ERIC BIDET

\section{Tableau 2}

Répartition par discipline universitaire

\begin{tabular}{|l|c|}
\hline \multicolumn{1}{|c|}{ Discipline } & Nombre de thèses \\
\hline Sciences de gestion & 6 \\
\hline Droit & 4 \\
\hline Economie & 3 \\
\hline Sociologie & 3 \\
\hline Sciences du langage & 1 \\
\hline Philosophie & 1 \\
\hline
\end{tabular}


Tableau 3

Répartition par objet de recherche

\begin{tabular}{|l|c|}
\hline \multicolumn{1}{|c|}{ Objet de recherche } & $\begin{array}{c}\text { Nombre } \\
\text { de thèses }\end{array}$ \\
\hline Santé - Action sociale - Education & 5 \\
\hline Autres coopératives & 4 \\
\hline $\begin{array}{l}\text { Coopération de crédit - } \\
\text { Finance solidaire }\end{array}$ & 3 \\
\hline Mutuelles d'assurance & 2 \\
\hline Associations & 1 \\
\hline Commerce équitable & 1 \\
\hline Transversal à l'ESS & 1 \\
\hline Autre & 1 \\
\hline
\end{tabular}

Tableau 4

Répartition géographique

\begin{tabular}{|l|c|}
\hline \multicolumn{1}{|c|}{ Zone géographique } & $\begin{array}{c}\text { Nombre } \\
\text { de thèses }\end{array}$ \\
\hline Paris & 9 \\
\hline Nord-Ouest & 3 \\
\hline Sud-Ouest & 2 \\
\hline Nord-Est & 1 \\
\hline Sud-Est & 1 \\
\hline $\begin{array}{l}\text { Etranger (Cameroun } \\
\text { et Luxembourg)* }\end{array}$ & 2 \\
\hline
\end{tabular}

* Notons que six de ces dix-huit thèses ont une dimension internationale (Amérique latine, Afrique, Europe). 